\title{
Artifacts and Visible Singularities in Limited Data X-Ray Tomography
}

\section{Quinto, Todd}

Published in:

Sensing and Imaging

Link to article, DOI:

10.1007/s11220-017-0158-7

Publication date:

2017

Document Version

Early version, also known as pre-print

Link back to DTU Orbit

Citation (APA):

Quinto, T. (2017). Artifacts and Visible Singularities in Limited Data X-Ray Tomography. Sensing and Imaging, 18(1). https://doi.org/10.1007/s11220-017-0158-7

\section{General rights}

Copyright and moral rights for the publications made accessible in the public portal are retained by the authors and/or other copyright owners and it is a condition of accessing publications that users recognise and abide by the legal requirements associated with these rights.

- Users may download and print one copy of any publication from the public portal for the purpose of private study or research.

- You may not further distribute the material or use it for any profit-making activity or commercial gain

- You may freely distribute the URL identifying the publication in the public portal

If you believe that this document breaches copyright please contact us providing details, and we will remove access to the work immediately and investigate your claim 


\title{
Artifacts and Visible Singularities in Limited Data $\mathrm{X}$-ray Tomography
}

\section{Eric Todd Quinto}

Received: date / Accepted: date

\begin{abstract}
We describe a principle to determine which features of an object will be easy to reconstruct from limited X-ray CT data and which will be difficult. The principle depends on the geometry of the data set, and it applies to any limited data set. We also describe a characterization of Frikel and the author explaining artifacts that can be added to limited angle reconstructions, and we provide an easy-to-implement method to decrease them. These ideas are justified using microlocal analysis, deep mathematics that involves Fourier theory. Reconstructions from simulated and real limited data are given to illustrate our ideas.
\end{abstract}

Keywords X-ray Tomography · Limited Data · Artifacts · Microlocal Analysis

\section{Introduction}

In this article, we introduce a mathematical principle that allows researchers to predict which object features will be visible and which will be invisible in reconstructions from limited X-ray tomographic data. The principle depends only on the geometry of the data set. We provide a second principle, depending on the data set and features of the object, that specifies when added streak artifacts can be created.

The author thanks the U.S. National Science Foundation for support under grant DMS 1311558 and the Otto Mønsteds Fond for its support during his time at Danish Technical University.

Eric Todd Quinto

Department of Mathematics, Tufts University

Tel.: +1-617-627-3402

Fax: +1-617-627-3966

E-mail: todd.quinto@tufts.edu

Present address: DTU Compute, Danish Technical University 
These principles will allow researchers to distinguish limitations of their algorithms from limitations in the data itself, and they suggest different data acquisition geometries depending on what type of features and shapes researchers want to image.

X-ray computed tomography $(\mathrm{CT})$ is an effective modality to find the densities of objects, and it has revolutionized diagnostic radiology and nondestructive evaluation. Typically, the object to be imaged is placed in a scanner and X-rays are taken over fairly uniformly distributed lines passing through all parts of the object. Such data are called complete tomographic data. Because the internal structure of an object is precisely shown in its CT reconstruction, radiologists can non-invasively detect tumors in the body, and engineers can assess the magnitude and nature of defects in industrial objects. However, there are cases in which a complete data set is not efficient to use or even possible to acquire. The data acquired in such cases are called limited tomographic data.

Tomography with limited data is more challenging than with complete data for several reasons. First, the standard tomographic algorithms $[35,36]$ do not apply without alteration because they require complete data. This alteration often adds artifacts to the reconstruction, as we will discuss in Sections 3, 5 and Remark 2. Furthermore, depending on the problem, it might be impossible to precisely determine the features of the object from limited data. Even when the object is uniquely determined by the data, reconstruction can be unstable and very sensitive to noise.

In $\S 2$, we describe the basic ideas and the first of our principles. In $\S 3$ we apply this to limited angle CT and give the second principle. In $\S 4$ we apply this to the exterior problem. Next, in $\S 5$ we describe a simple method to suppress the added artifacts that can occur in limited data problems. Finally, in $\S 6$ we provide a basic description of microlocal analysis, the mathematics behind our ideas.

The microlocal analysis we use is based on $[18,22,23]$ and the principle for visible and invisible singularities is based on our analysis in [42]. The description of added artifacts is from [14]. Related theoretical work is in [2,11, $17,16,21,39]$ and other references which will be given in the sections.

\section{The Basics}

We now introduce notation then describe our first principle. The X-ray transform integrates over lines, and we use the parallel beam parameterization for lines. For $\varphi \in[-\pi, \pi]$ and $p \in \mathbb{R}$

$$
L(\varphi, p)=\left\{x \in \mathbb{R}^{2} \mid x \cdot \bar{\theta}=p\right\} \quad \text { where } \bar{\theta}=\bar{\theta}(\varphi):=(\cos (\varphi), \sin (\varphi))
$$

is the line perpendicular to $\bar{\theta}(\varphi)$ and through the point $p \bar{\theta}(\varphi)$. Because $x \cdot \bar{\theta}(\varphi)=-(x \cdot \bar{\theta}(\varphi+\pi))$, each line can be parameterized in two ways:

$$
L(\varphi, p)=L(\varphi+\pi,-p) .
$$




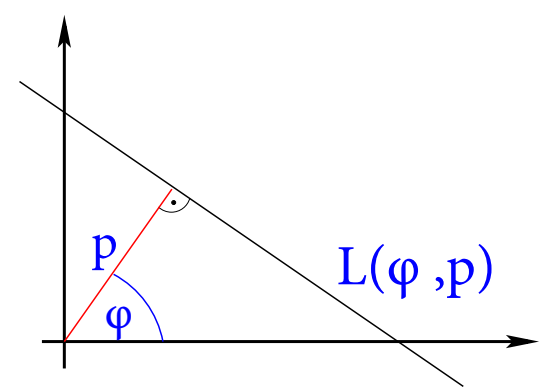

Fig. 1: The line $L(\varphi, p)$.

Let $f$ be the density function of the object to be reconstructed. Using Beer's Law [35], the measured data from an X-ray CT scanner provides the $X$-ray transform of $f$ (assuming monochromatic X-rays):

$$
R f(\varphi, p)=\int_{x \in L(\varphi, p)} f(x) d s
$$

where $d s$ is standard arc length measure. The complete data backprojection operator of the function $g=g(\varphi, p)$ is

$$
R^{*} g(x)=\int_{\varphi=-\pi}^{\pi} g(\varphi, x \cdot \bar{\theta}(\varphi)) d \varphi .
$$

Since, for all $\varphi, x \in L(\varphi, x \cdot \bar{\theta}(\varphi)), R^{*} g(x)$ is the integral of $g$ over all lines through $x$. The filtered backprojection (FBP) reconstruction of a function $f$ from complete data is

$$
\begin{gathered}
f=\frac{1}{4 \pi} R^{*} \Lambda R f \quad \text { where } \\
\Lambda g(\varphi, p)=\frac{1}{2 \pi} \int_{\tau=-\infty}^{\infty} \int_{s=-\infty}^{\infty} e^{i \tau(p-s)}|\tau| g(\varphi, s) d s d \tau
\end{gathered}
$$

In general, an approximation to $\Lambda$ is used with a filter that has Fourier transform an approximation to the function $|\tau|[35,36]$.

The features we consider are described by boundaries between parts of objects, for example, between the heart and chest cavity in the body. Mathematically, we consider density functions $f$ that are smooth except for jump discontinuities along piecewise smooth curves-the boundaries of the organs and bones in the body. We will call such functions piecewise smooth.

In fact, when one looks at an X-ray picture, such as the left picture in Figure 2, the edges (boundaries) of the bones are the easiest features to see. In the right picture of that figure, the disk "represents" the cross-section of a bone and one sees that the data change the most (has infinite slope) at tangent lines to the disk. This is what the eye interprets as a boundary. 

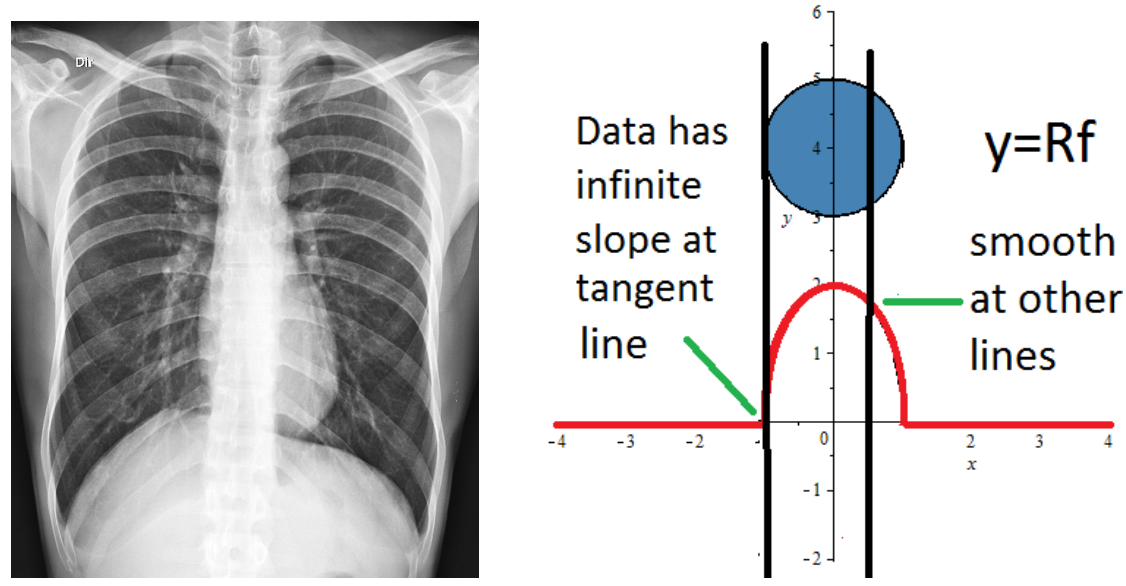

Fig. 2: Left: Chest X-ray (pictures.google.com) Right: graph of the Radon transform of a disk over vertical lines. Note how boundaries are the most prominent features in the left picture. In the right graph, the data are not smooth (have infinite slope) at the tangent lines to the disk but the data are smooth at other lines.

Recall that in limited data tomography, data over some lines are missing. Furthermore, the features we want to reconstruct are boundaries of parts of the body. The analysis in the last paragraph suggests that if a line in the data set is tangent to a boundary, that part of the boundary should be easy to reconstruct. It also suggests that, if there is no line in the data set tangent to a boundary, that boundary will be difficult to reconstruct. This leads to the following.

Principle 1 ([42]) (a) If a boundary of a feature of the body is tangent to a line in a limited data set, then that boundary should be easy to reconstruct from that limited data. Such boundaries are called visible boundaries (from this limited data).

(b) If a boundary is not tangent to any line in a limited data set, then that boundary should be difficult to reconstruct from the limited data. Such boundaries are called invisible boundaries (from this limited data).

Principle 1 will be generalized and justified in $\S 6$.

\section{Limited Angle CT}

In this section, we apply this principle to limited angle tomography, and we present another principle that will explain added artifacts.

Note that, by $(2)$, each line is parameterized by $(\varphi, p)$ for some $\varphi \in[-\pi / 2, \pi / 2]$ and $p \in \mathbb{R}$. In limited angle $X$-ray $C T$, the scanner cannot take 
data all around the object, and so the data are in some set $(\varphi, p) \in[-\alpha, \alpha] \times \mathbb{R}$ where $\alpha<\pi / 2$. This is a limited data set because any lines not parameterized by angles in $[-\alpha, \alpha]$ are not in the data set. Our arguments apply to arbitrary limited angle data sets with $\varphi \in[a, b]$ with $b-a<\pi$ by rotating the plane.

Much research has been done on limited angle CT, e.g., [5,31] including analysis of artifacts and visible singularities [14,24,25,37,42] and algorithms using this information [12]. Limited angle ROI CT (i.e., data for $|\varphi|<\alpha$ and $|p|<r$ for some $r>0$ ) is considered [29,46] and limited angle exterior CT (data for $|\varphi|<\alpha$ and $|p|>r$ for some $r>0$ ) is addressed in [43,45].

Let $\alpha \in(0, \pi / 2)$ and define the limited angle backprojection operator

$$
R_{\alpha}^{*} g(x)=\int_{\varphi=-\alpha}^{\alpha} g(\varphi, x \cdot \bar{\theta}(\varphi)) d \varphi .
$$

Note that $R_{\alpha}^{*}$ uses only limited angle data for angles $\varphi \in[-\alpha, \alpha]$. A standard reconstruction method is to replace $R^{*}$ in (4) by $R_{\alpha}^{*}$ to get a reconstruction operator suitable for limited angle data:

$$
\mathcal{L}_{\alpha} f=\frac{1}{4 \pi} R_{\alpha}^{*} \Lambda R f
$$

where $\Lambda$ is given by (4). Of course, $\mathcal{L}_{\alpha}$ does not reconstruct $f$ and we will see what it does reconstruct in the next examples.

Example 1 Figure 3, gives a reconstruction using the limited angle FBP operator $\mathcal{L}_{\alpha}(6)$ for angles between $-\pi / 4$ and $\pi / 4(\alpha=\pi / 4)$.
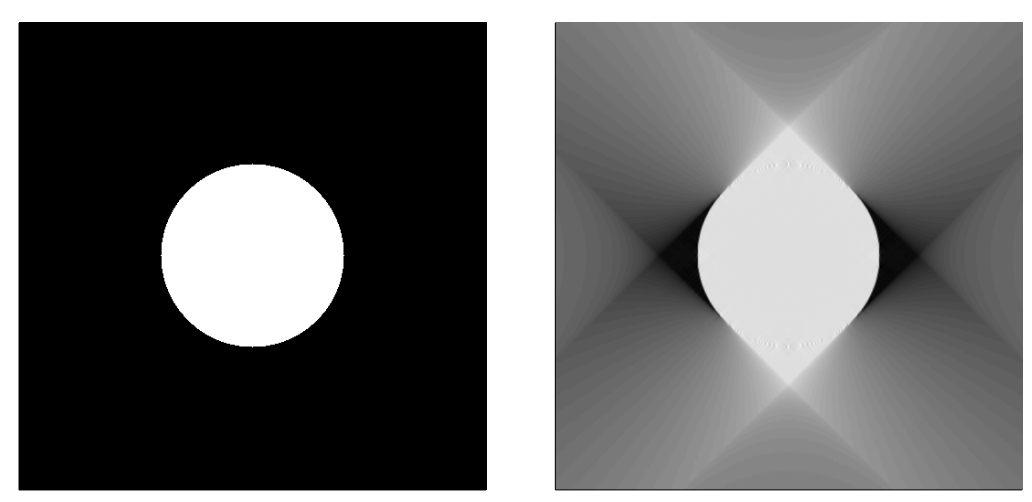

Fig. 3: Left: disk phantom, Right: Limited angle FBP reconstruction [14] using data for angles $\varphi \in[-\pi / 4, \pi / 4]$ (C) IOP Publishing. Reproduced by permission of IOP Publishing. All rights reserved)

Figure 3 illustrates Principle 1. The lines in the data set are generally vertical and the vertical boundaries are visible (i.e., well-reconstructed); some line in the data set is tangent to each part of the boundary that is visible in the 
reconstruction. Furthermore, the invisible boundaries of the disk are tangent to generally horizontal lines (line of slope between -1 and +1 ), and these lines are not in the data set.

Note that there are strong artifacts on four lines tangent to the circle: two lines with $\varphi=-\pi / 4$ (i.e., with slope +1 ) and two lines with $\varphi=\pi / 4$ (i.e., with slope -1$)$. This leads us to our second principle.

Principle 2 For the limited angle problem for $\varphi \in[-\alpha, \alpha]$, artifacts can occur on lines at the end of the data set-for $\varphi=-\alpha$ or $\varphi=+\alpha$. Artifact lines will be tangent to the boundary of some feature in the object.

Principle 2 was proven in work with Frikel [14] and a refinement of this principle is valid for many other tomographic problems $[15,38]$.

Example 2 Now, we consider the limited angle reconstruction of a brain over the same set of lines as Example $1(\alpha=\pi / 4)$.
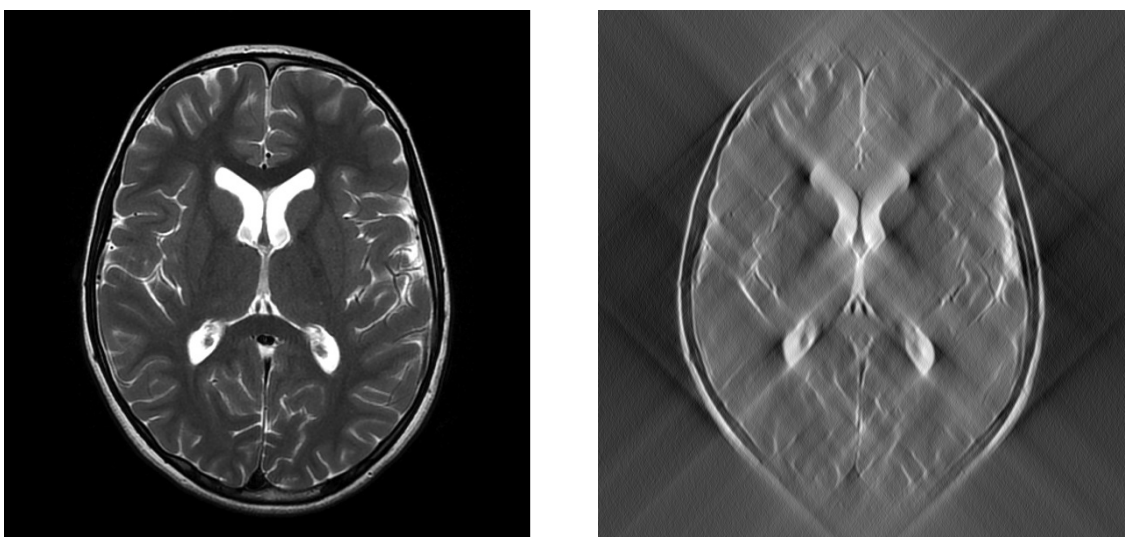

Fig. 4: Left: Brain phantom [radiopedia.org]. Right: FBP reconstruction [14] using limited angle data for angles $\varphi \in[-\pi / 4, \pi / 4]$ (C) IOP Publishing. Reproduced by permission of IOP Publishing. All rights reserved).

Principle 1 is illustrated in Figure 4 because the generally vertical boundaries are well-reconstructed, but horizontal boundaries are invisible. Principle 2 is illustrated by the strong artifact lines with slope -1 or +1 (lines at the ends of the data set) that are tangent to features of the brain.

Our examples and the principles show that, if the object has horizontal boundaries, one should not use limited angle data for $\varphi \in[-\alpha, \alpha]$. If possible, one could rotate the scanner so the limited data were, for example in $[\pi / 2-\alpha, \pi / 2+\alpha]$, but then one would miss vertical boundaries. Alternatively, one might consider reconstruction methods that extend data in the range of 
the Radon transform $[1,30]$ or ones that use a priori information if the object is piecewise constant or otherwise sparse $[9,13,29]$ or test algebraic reconstruction methods and regularization $[20,32,34]$.

\section{Exterior CT}

In this section, we apply our principles to the exterior problem in X-ray CT. In this case, the data are over lines $L(\varphi, p)$ for $\varphi \in[-\pi, \pi]$ and for $p>r$ for some fixed $r>0$. This describes the set of lines $L(\varphi, p)$ that are outside the disk of radius $r$ centered at the origin. Standard reconstruction methods are not usable for exterior data; for example, FBP or limited angle FBP requires data $R f(\varphi, s)$ for all $s \in \mathbb{R}$ to evaluate $\Lambda R f$ in (4).

Cormack [3] provided a reconstruction method for exterior data, but it was unstable and he subsequently developed a different reconstruction method using complete data [4]. Natterer [34] developed an exterior reconstruction method using regularization. The reconstructions in this section are from the author's algorithms in $[40,41,45]$.

To show the versatility of Principle 1, we use it for two different data sets that are reconstructed with two different algorithms.

Example 3 This example illustrates Principle 1, but it also shows how limitations in the algorithm can affect the reconstruction.

The author developed a singular value decomposition (SVD) reconstruction method for the exterior problem in [40] and tested it on a mathematical phantom in [41]. The phantom in Figure 5 is similar to the one in [34] but more difficult for the author's algorithm than Natterer's phantom.
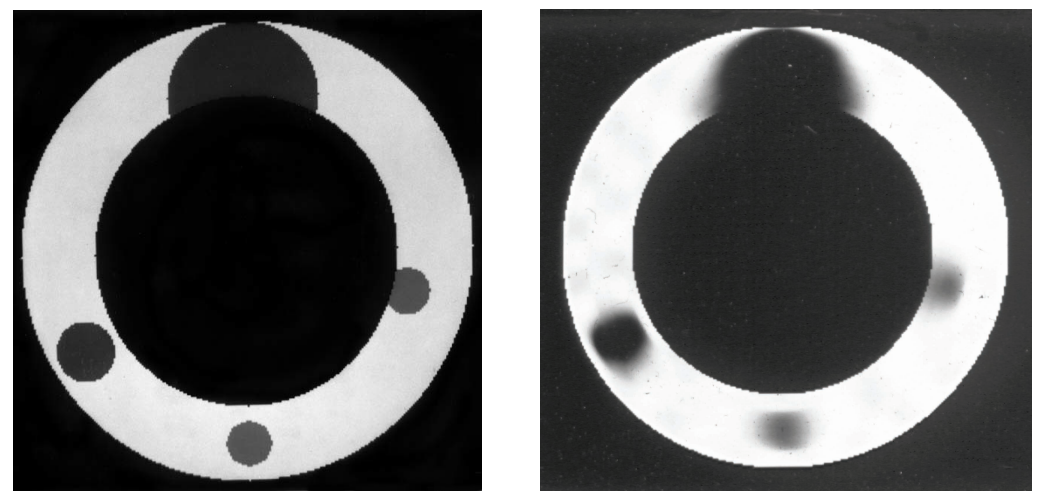

Fig. 5: Left: phantom. Right: reconstruction using the algorithm in [41] and with slightly less than $1 \%$ noise. The region being reconstructed is the annulus between radius $r=1$ and $r=1.5$ (C) IOP Publishing. Reproduced by permission of IOP Publishing. All rights reserved). 
In [16], we justify Principle 1 for reconstruction methods based on backprojection, but it is valid more generally. Reconstruction of invisible boundaries is highly ill-posed since the forward operator, $R$, smooths them out in the limited data (see Theorem 1 part (b) and Remark 1). Although the reconstruction method used on this phantom does not use backprojection, the reconstruction in Figure 5 is consistent with Principle 1: the boundaries tangent to lines in the data set-the inside and outside boundaries of the small disks-are better reconstructed than the "side" boundaries-ones that are not tangent to any lines in the data set.

Even if visible boundaries should always be reconstructed better than invisible ones, the algorithm itself determines how they are reconstructed. Comparing the reconstruction of the disk in Figure 3 using limited angle FBP with the reconstruction using the SVD in Figure 5, one sees that the visible singularities are much sharper in the limited angle FBP reconstruction. However, the invisible boundaries are not at all visible in that reconstruction, but they are visible, if rather fuzzy, in the reconstruction in Figure 5.

Some effects are not directly related to the principles but to the algorithm itself. In Figure 5, the inner boundaries in the disks at 6 o'clock and 7:30 o'clock which are visible are sharp but slightly distorted. The noise added to the data causes the slight splotches in the light annulus outside the little disks. These are both effects of the algorithm (which uses polar Fourier series) and the general ill-posedness of the exterior problem and not a direct consequence of the principles.

Example 4 In this example, we evaluate the reconstruction of real data of a large, solid-filled cylindrical vessel with crack simulations. The region to be reconstructed is the outer annulus of this object, with radius between $r=34.75$ and $r=36.75 \mathrm{~cm}$. Furthermore, because of a data acquisition problem, limited angle exterior data were given; data were over lines $L(\varphi, p)$ for $\varphi \in[0,3 \pi / 4]$ and $p \in[34.75,36.75]$. The object was designed to simulate a large rocket and was done in a project with Perceptics, Inc.

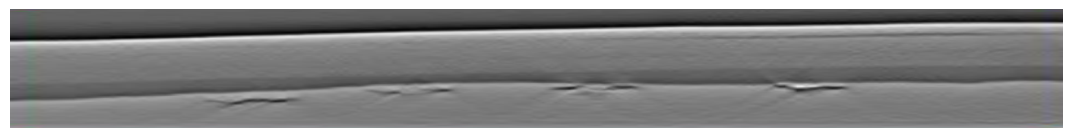

Fig. 6: Reconstruction using the algorithm in [45] of a large, solid-filled cylindrical vessel with crack simulations. The limited angle exterior data are for angles $\varphi \in[0,3 \pi / 4]$ and $p \in[34.7536 .75] \mathrm{cm}$. The picture is a quarter of the annulus graphed in polar coordinates $(r, \varphi)$ (where Cartesian coordinate $x=(r \cos (\varphi), r \sin (\varphi)))$. Angles $\varphi \in[0, \pi / 2]$ are along the horizontal axis and radius $r \in[34.75,36.75]$ on the vertical axis, with the inside at the bottom and the outside at the top. The outer boundary of the object is the dark curve near the top of the reconstruction. The scale in $r$ is magnified so the cracks are easier to see. 
X-rays cannot penetrate the central core of large objects such as rocket bodies, and only data in the outer annulus is usable. The author developed an improved version of his SVD algorithm in [43] that gives better reconstructions than the original ones in [41]. Subsequently, he developed a local exterior reconstruction algorithm in [45] that is based on Lambda Tomography-a type of filtered back projection $[7,8,50]$ but adapted for limited angle exterior data (see also [33] for another outlook). This algorithm gave slightly clearer reconstructions.

The defects in the object are all along arcs that are tangent to lines in the exterior data set so, by Principle 1, they are easy to reconstruct from exterior data.

These last two examples and Principle 1 show that exterior tomography is good for detecting boundaries that are generally along a circular arc (roughly centered at the center of the object) since such boundaries are tangent to lines in the exterior data set. Many potential defects in rocket shells are on such arcs, such as cracks or separations between stages of the rocket. Therefore, exterior CT can be useful for the NDE of rockets shells.

Exterior CT might not be as effective if the features or cracks were generally going from the inside of the annulus out since they would have invisible boundaries as they are not tangent to lines in the exterior data set.

\section{Artifact Suppression}

We now give a simple artifact suppression technique that applies to a broad range of tomographic problems. The artifacts are caused by the sharp cutoff in data at the end of the data set, as we discuss in Remark 2, and the solution is to replace that with a soft cutoff by multiplying by a smooth cutoff function. This is proven in [14] for limited angle CT and [15] in for a broad range of tomographic modalities.

The technique will now be described for limited angle CT with data for $\varphi \in[-\alpha, \alpha]$. One chooses a function $\psi(\varphi)$ that is zero except on the open interval $(-\alpha, \alpha)$, is equal to one on most of $(-\alpha, \alpha)$ and has a smooth $\left(C^{\infty}\right)$ transition between 0 and 1 near the endpoints $-\alpha$ and $\alpha$. Then, one uses the artifact-compensated limited angle reconstruction operator

$$
\mathcal{L}_{\alpha, \psi} f=R_{\alpha}^{*} \Lambda(\psi R f) .
$$

In [14], we prove that this operator does not add artifacts and shows most of the visible singularities described in Principle 1 . Our proof shows that $\mathcal{L}_{\alpha, \psi}$ is a pseudodifferential operator [22] that is elliptic for most of the visible singularities. In [24], non-smooth cutoffs are analyzed.

Figure 7 illustrates the effect of the artifact suppression. Streaks are clearly evident in the middle reconstruction without artifact suppression. They are decreased in the right-hand reconstruction which uses moderate artifact reduction. However, there is a slight loss of detail for visible boundaries that are 

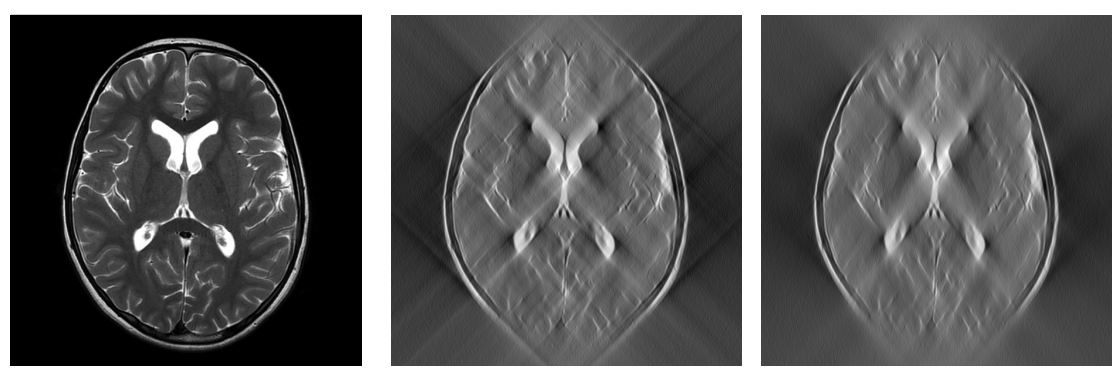

Fig. 7: Left: Brain phantom. [radiopedia.org] Middle: FBP reconstruction [14] without artifact reduction. Right: FBP reconstruction with moderate artifact reduction (C) IOP Publishing. Reproduced by permission of IOP Publishing. All rights reserved)

tangent to lines near then ends of the data set (i.e., tangent to lines $L(\varphi, p)$ with $\varphi$ close to $\pm \alpha$ ) since the cutoff $\psi$ de-emphasizes data for $\varphi$ near $\pm \alpha$.

To use this method on other tomographic data sets, one just chooses the function $\psi$ to be equal to one on most of the data set and smoothly transition to zero at the boundary of the data set and be zero outside the data set.

In general, if one extends the data from a limited data set, one should extend without sharp transitions or jumps at the end of the data set. Having a sharp transition, such as a jump, at the boundary of the data set can create extra artifacts in the reconstruction.

For the exterior problem, there can be added artifacts, but both reconstruction methods used in $\S 4$ include smoothing steps that suppress them.

\section{Basic Microlocal Analysis}

We now provide the mathematical background to make our principles and ideas rigorous and general.

Microlocal analysis $[22,23,49]$ provides a precise characterization of singularities of functions as well as a rigorous description of what operators, including $R, R^{*}$, and $R_{\alpha}^{*}$ do to those singularities. Basic discussions of microlocal analysis in tomography are in $[27,42]$ and microlocal analysis is used in more general settings (e.g., [17]), seismics (e.g., [6]) radar (e.g., [26,48]) and X-ray CT (e.g., [11, 25, 28,37]).

The fundamental idea is the wavefront set, a rigorous and general description of singularities. It is defined in terms for the Fourier transform on $\mathbb{R}^{2}$,

$$
\mathcal{F} f(y)=\frac{1}{2 \pi} \int_{x \in \mathbb{R}^{2}} e^{-i x \cdot y} f(x), d x
$$

The inverse Fourier transform of an integrable function is continuous [47]. Using this and derivative rules for the Fourier transform, one can prove that, if 
$\mathcal{F} f$ is rapidly decaying at infinity (decaying faster than any power of $1 /\|y\|$ ) then $f$ is $C^{\infty}$ smooth. This is a global condition: global rapid decay of $\mathcal{F} f$ implies global smoothness of $f$. The wavefront set localizes and microlocalizes this connection between smoothness of $f$ and rapid decay of its Fourier transform.

Definition 1 Let $f$ be a function on $\mathbb{R}^{2}$ and let $x_{0} \in \mathbb{R}^{2}$ and $\xi_{0} \in \mathbb{R}^{2} \backslash \mathbf{0}$. Then, we say that $f$ is smooth at $x_{0}$ in direction $\xi_{0}$ if there is a smooth function $\phi \in C_{c}^{\infty}\left(\mathbb{R}^{2}\right)$ such that $\phi\left(x_{0}\right) \neq 0$ and an open cone $V$ containing $\xi_{0}$ such that given any $N \in \mathbb{N}$, there is a $C_{N}$ such that

$$
|\mathcal{F}(\phi f(y))| \leqslant C_{N}(1+\|y\|)^{-N} \text { for } y \in V .
$$

If $f$ is not smooth at $x_{0}$ in direction $\xi_{0}$ then $\left(x_{0}, \xi_{0}\right) \in \mathrm{WF}(f)$, the wavefront set of $f$.

The cutoff function $\phi$ localizes $f$ around $x_{0}$, and the direction $\xi_{0}$ "microlocalizes" by focusing on whether the localized Fourier transform $\mathcal{F}(\phi f)$ is rapidly decaying in some open cone $V$ containing $\xi_{0}$.

Example 5 If the function $f$ is equal to 1 inside a set $\Omega$ with smooth boundary and equal to 0 outside, then $\mathrm{WF}(f)$ is the set of normals to the boundary of $\Omega$, as illustrated in Figure 8 .

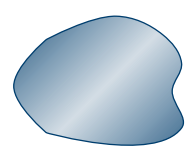

$f$ is $=1$ inside this set $=0$ outside the set

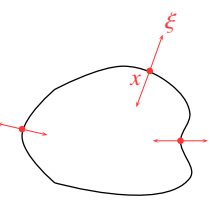

Normals to the boundary $(x, \xi)$ are in $\mathrm{WF}(f)$

Fig. 8: Left: The set $\Omega$. Right: $\mathrm{WF}(f)$ where $f$ is the function equal to 1 on $\Omega$ and zero elsewhere.

This is true more generally: if $f$ is piecewise smooth, then $\mathrm{WF}(f)$ is the set of normals to the boundary curves at the jump singularities of $f$.

For piecewise smooth functions, there is a direct correspondence between wavefront set and tangent lines to the boundary curves. If $f$ is such a function then $\left(x_{0}, \xi_{0}\right) \in \mathrm{WF}(f)$ if $x_{0}$ is a point on one of those boundary curves and $\xi_{0}$ is normal to that boundary at $x_{0}$. Let $L_{0}$ be the tangent line to that boundary 
at $x_{0}$. Since, $\xi_{0}$ is normal to the boundary at $x_{0}$, it is normal to the tangent line $L_{0}$. Thus $\left(x_{0}, \xi_{0}\right) \in \mathrm{WF}(f)$ exactly when the line through $x_{0}$ perpendicular to $\xi_{0}$ is tangent to the boundary curve at $x_{0}$.

Our next theorem helps justify Principle 1.

Theorem 1 (Microlocal Regularity Theorem for $R$ [42]) Singularities of $f$ produce singularities of $R f$. Let $L_{0}=L\left(\varphi_{0}, p_{0}\right)$ be a line in the plane. Let $x_{0} \in L_{0}$ and let $\xi_{0}$ be a normal vector to $L_{0}$. Assume $\left(x_{0}, \xi_{0}\right) \in W F(f)$.

(a) The singularity of $f$ at $\left(x_{0}, \xi_{0}\right)$ will cause a unique corresponding singularity $\lambda \in W F(R f)$, and that singularity will be at $\left(\varphi_{0}, p_{0}\right)$.

(b) If $L_{0}$ is not in a limited data set, then the singularities of $f$ normal to $L_{0}$ will not affect smoothness of the limited data (i.e., the part of WF(Rf) above the limited data set will be the same whether $f$ has wavefront set normal to $L_{0}$ or not).

This theorem is true because $R$ is a Fourier integral operator (e.g., [19, 44]) and these operators transform wavefront sets in precise ways [22]. The singularity $\lambda$ is explicitly given in [42].

Remark 1 We now use Theorem 1 to partially justify Principle 1 in general. Let $f$ be a function and assume $\left(x_{0}, \xi_{0}\right) \in \mathrm{WF}(f)$. Let $L_{0}$ be the line through $x_{0}$ normal to $\xi_{0}$. Choose $\varphi_{0}$ and $p_{0}$ such that $L_{0}=L\left(\varphi_{0}, p_{0}\right)$. By Theorem 1 part (a), there will be a unique singularity in $\mathrm{WF}(R f)$ caused by the singularity of $f$ at $\left(x_{0}, \xi_{0}\right)$ and it is at $\left(\varphi_{0}, p_{0}\right)$. Therefore, if $L_{0}$ is in a limited data set, this singularity of $f$ at $\left(x_{0}, \xi_{0}\right)$ will be visible in that limited data. However, if $L_{0}$ is not in the data set, then, that singularity is not visible in the data and by part (b), that singularity does not affect smoothness of the data $R f$ on that limited data set.

This partially justifies Principle 1 by explaining how singularities of $f$ affect $R f$ on a limited data set. What is missing is the connection with the reconstruction. That is provided for filtered back projection type reconstruction methods in [16] using properties of the Fourier integral operator $R^{*}$.

By Theorem 1 part (b), singularities of $f$ that are not normal to lines in the data set have no effect on singularities of $R f$ on that limited data set. By an argument in [10] starting with this observation, reconstruction of those singularities is discontinuous in any range of Sobolev norms. This is one way to say that recovery of the invisible boundaries in Principle 1 is, in general, much more difficult than for visible boundaries. Also, the principle is reflected in all our reconstructions.

We now specialize to limited angle tomography for angles $\varphi \in[-\alpha, \alpha]$ where $\alpha$ is chosen in $(0, \pi / 2)$. Let $\left(x_{0}, \xi_{0}\right) \in \mathrm{WF}(f)$. We say that $\left(x_{0}, \xi_{0}\right)$ is a visible singularity of $f$ (for $\mathcal{L}_{\alpha}$ ) if $\xi_{0}$ is parallel $\bar{\theta}(\varphi)$ for some $\varphi \in(-\alpha, \alpha)$-that is $\xi_{0}$ is normal to a line in the limited angle data set that goes through $x_{0}$. We say $\left(x_{0}, \xi_{0}\right)$ is an invisible singularity of $f$ if $\xi_{0}$ is not parallel $\bar{\theta}(\varphi)$ for any $\varphi \in[-\alpha, \alpha]$. The case $\varphi= \pm \alpha$ is special; singularities parallel $\bar{\theta}( \pm \alpha)$ can be added or masked. 
Theorem 2 (Microlocal Regularity Theorem for $\mathcal{L}_{\alpha}$ ) Let $\alpha \in(0, \pi / 2)$ and let $\mathcal{L}_{\alpha}$ be the limited angle FBP operator in (6). Let $f$ be a function of compact support and $\left(x_{0}, \xi_{0}\right) \in W F(f)$.

1. If $\left(x_{0}, \xi_{0}\right)$ is a visible singularity of $f$ then $\left(x_{0}, \xi_{0}\right) \in W F\left(\mathcal{L}_{\alpha} f\right)$.

2. If $\left(x_{0}, \xi_{0}\right)$ is an invisible singularity of $f$ then $\left(x_{0}, \xi_{0}\right) \notin W F\left(\mathcal{L}_{\alpha} f\right)$.

Theorem 2 follows from [16, Corollary 4.1, equation (4.9)].

This theorem justifies the definition of visible and invisible singularity for $\mathcal{L}_{\alpha}$. A visible singularity of $f$ will be visible in the reconstruction $\mathcal{L}_{\alpha} f$, and an invisible singularity of $f$ will not be visible in the reconstruction. For $\mathcal{L}_{\alpha}$ and piecewise smooth functions, this theorem is equivalent to Principle 1 by the correspondence between tangent lines to boundary curves and wavefront set perpendicular to those curves.

Remark 2 We now justify Principle 2 for $\mathcal{L}_{\alpha}$. The limited angle backprojection (5) can be written $R_{\alpha}^{*} g=R^{*}(\chi g)$ where $\chi(\varphi, p)$ is equal to one if $\varphi \in[-\alpha, \alpha]$ and zero otherwise. Now, $(\chi \Lambda R f)$ will be zero off of the limited angle data set but, in general, nonzero on the data set. This can create an extra jump singularity in $(\chi \Lambda R f)$, and this can help produce the extra artifacts in $\mathcal{L}_{\alpha} f=R^{*}(\chi \Lambda R f)$ described in Principle 2. This is proven in generality in [16, Theorem 5.4 and equation (5.10)].

Acknowledgements The author is indebted to DTU Compute for a stimulating and congenial semester. He thanks his DTU colleagues, graduate students, and Danish high school students with whom he discussed these ideas for their astute questions and observations about this work. The author thanks Jürgen Frikel for discussions and work that informed this article as well as for Figure 8. He thanks Leise Borg and Jakob Jørgensen for their insights on limited data tomography. The author also thanks Ming Jiang for helpful discussions about this research. He thanks the local organizers for a stimulating International Conference on Sensing and Imaging, 2016 in Taiyuan, China.

\section{References}

1. Bates, R., Lewitt, R.: Image reconstruction from projections: I: General theoretical considerations, II: Projection completion methods (theory), III: Projection completion methods (computational examples). Optik 50, I: 19-33, II: 189-204, III: 269-278 (1978)

2. Beylkin, G.: The inversion problem and applications of the generalized Radon transform. Comm. Pure Appl. Math. 37, 579-599 (1984)

3. Cormack, A.M.: Representation of a function by its line integrals with some radiological applications. J. Appl. Physics 34, 2722-2727 (1963)

4. Cormack, A.M.: Representation of a function by its line integrals with some radiological applications II. J. Appl. Physics 35, 2908-2913 (1964)

5. Davison, M., Grünbaum, F.: Tomographic Reconstruction with Arbitrary Directions. Comm. Pure Appl. Math. 34, 77-120 (1981)

6. deHoop, M.: Microlocal analysis of seismic inverse scattering, Math. Sci. Res. Inst. Publ., vol. 47, pp. 219-296. Cambridge Univ. Press, Cambridge, England (2003)

7. Faridani, A., Finch, D., Ritman, E.L., Smith, K.T.: Local tomography, II. SIAM J. Appl. Math. 57, 1095-1127 (1997)

8. Faridani, A., Ritman, E.L., Smith, K.T.: Local tomography. SIAM J. Appl. Math. 52, 459-484 (1992) 
9. Fidler, T., Grasmair, M., Scherzer, O.: Shape Reconstruction with A Priori Knowledge Based on Integral Invariants. SIAM J. Imaging Sciences 5(2), 726-745 (2012)

10. Finch, D.V.: Uniqueness for the attenuated X-ray transform in the physical range. SIAM J. Appl. Math. pp. 197-203 (1986)

11. Finch, D.V., Lan, I.R., Uhlmann, G.: Microlocal Analysis of the Restricted X-ray Transform with Sources on a Curve. In: G. Uhlmann (ed.) Inside Out, Inverse Problems and Applications, MSRI Publications, vol. 47, pp. 193-218. Cambridge University Press (2003)

12. Frikel, J.: Sparse regularization in limited angle tomography. Applied and Computational Harmonic Analysis 34(1), 117-141 (2013)

13. Frikel, J.: Sparse regularization in limited angle tomography. Applied and Computational Harmonic Analysis 34(1), 117-141 (2013)

14. Frikel, J., Quinto, E.T.: Characterization and reduction of artifacts in limited angle tomography. Inverse Problems 29(12), 125,007 (2013)

15. Frikel, J., Quinto, E.T.: Artifacts in incomplete data tomography with applications to photoacoustic tomography and sonar. SIAM J. Appl. Math. 75(2), 703-725 (2015)

16. Frikel, J., Quinto, E.T.: Limited data problems for the generalized Radon transform in $\mathbb{R}^{n}$. SIAM J. Math. Anal. 48, 2301-2318 (2016)

17. Greenleaf, A., Uhlmann, G.: Non-local inversion formulas for the X-ray transform. Duke Math. J. 58, 205-240 (1989)

18. Guillemin, V.: On some results of Gelfand in integral geometry. Proceedings Symposia Pure Math. 43, 149-155 (1985)

19. Guillemin, V., Sternberg, S.: Geometric Asymptotics. American Mathematical Society, Providence, RI (1977)

20. Hansen, P.C.: Discrete Inverse Problems: Insight and Algorithms. SIAM, Philadelphia, USA (2010). (213 pages)

21. Helgason, S.: Integral geometry and Radon transforms. Springer, New York (2011)

22. Hörmander, L.: Fourier Integral Operators, I. Acta Mathematica 127, 79-183 (1971)

23. Hörmander, L.: The analysis of linear partial differential operators. I. Classics in Mathematics. Springer-Verlag, Berlin (2003). Distribution theory and Fourier analysis, Reprint of the second (1990) edition [Springer, Berlin]

24. Katsevich, A.: Local tomography for the limited-angle problem. J. Math. Anal. Appl. 213(1), 160-182 (1997)

25. Katsevich, A.: Reconstruction algorithms for a class of restricted ray transforms without added singularities. J. Fourier Anal. Appl. p. (22p.) (2016). DOI 10.1007/s00041-0169473-y

26. Krishnan, V.P., Quinto, E.T.: Microlocal aspects of common offset synthetic aperture radar imaging. Inverse Probl. Imaging 5(3), 659-674 (2011). DOI 10.3934/ipi.2011.5.659

27. Krishnan, V.P., Quinto, E.T.: Microlocal Analysis in Tomography. In: O. Scherzer (ed.) Handbook of Mathematical Methods in Imaging, chap. 18, pp. 847-902. Springer Verlag, New York, Berlin (2015)

28. Kuchment, P., Lancaster, K., Mogilevskaya, L.: On local tomography. Inverse Problems 11, 571-589 (1995)

29. Liu, B., Wang, G., Ritman, E.L., Cao, G., Lu, J., Zhou, O., Zeng, L., Yu, H.: Image reconstruction from limited angle projections collected by multisource interior x-ray imaging systems. Physics in Medicine and Biology 56, 6337-6357 (2011). DOI 10.1088/00319155/56/19/012

30. Louis, A.K.: Picture Reconstruction from Projections in Restricted Range. Mathematical Methods in the Applied Sciences 2, 209-220 (1980)

31. Louis, A.K.: Incomplete data problems in X-ray computerized tomography I. Singular value decomposition of the limited angle transform. Numerische Mathematik 48, 251$262(1986)$

32. Louis, A.K.: A unified approach to regularization methods for linear ill- posed problems. Inverse Problems 15, 489-498 (1999)

33. Louis, A.K.: Feature reconstruction in inverse problems. Inverse Problems 27, 065,010 (2011)

34. Natterer, F.: Efficient implementation of 'optimal' algorithms in computerized tomography. Math. Methods in the Appl. Sciences 2, 545-555 (1980) 
35. Natterer, F.: The mathematics of computerized tomography. B. G. Teubner, Stuttgart (1986)

36. Natterer, F., Wübbeling, F.: Mathematical methods in image reconstruction. SIAM Monographs on Mathematical Modeling and Computation. Society for Industrial and Applied Mathematics (SIAM), Philadelphia, PA (2001)

37. Nguyen, L.V.: How strong are streak artifacts in limited angle computed tomography? Inverse Problems 31(5), 055,003, 26 (2015)

38. Nguyen, L.V.: On artifacts in limited data spherical radon transform: flat observation surfaces. SIAM J. Math. Anal. 47(4), 2984-3004 (2015). DOI 10.1137/140980740. URL http://dx.doi.org/10.1137/140980740

39. Palamodov, V.: Reconstruction from Limited Data of Arc Means. J. Fourier Anal. Appl. 6, 25-42 (2000)

40. Quinto, E.T.: Singular value decompositions and inversion methods for the exterior Radon transform and a spherical transform. J. Math. Anal. Appl. 95, 437-448 (1983)

41. Quinto, E.T.: Tomographic reconstructions from incomplete data-numerical inversion of the exterior Radon transform. Inverse Problems 4, 867-876 (1988)

42. Quinto, E.T.: Singularities of the X-ray transform and limited data tomography in $\mathbb{R}^{2}$ and $\mathbb{R}^{3}$. SIAM J. Math. Anal. 24(5), 1215-1225 (1993)

43. Quinto, E.T.: Exterior and Limited Angle Tomography in Non-destructive Evaluation. Inverse Problems 14, 339-353 (1998)

44. Quinto, E.T.: An introduction to X-ray tomography and Radon transforms. In: The Radon transform, inverse problems, and tomography, pp. 1-23. Amer. Math. Soc., Providence, RI (2006)

45. Quinto, E.T.: Local Algorithms in Exterior Tomography. Journal of Computational and Applied Mathematics 199, 141-148 (2007)

46. Quinto, E.T., Öktem, O.: Local tomography in electron microscopy. SIAM J. Appl. Math. 68, 1282-1303 (2008)

47. Rudin, W.: Functional analysis. McGraw-Hill Book Co., New York (1973). McGraw-Hill Series in Higher Mathematics

48. Stefanov, P., Uhlmann, G.: Is a curved flight path in SAR better than a straight one? SIAM J. Appl. Math. 73(4), 1596-1612 (2013)

49. Trèves, F.: Introduction to Pseudodifferential and Fourier Integral Operators. Volume 2: Fourier Integral Operators. Plenum Press, New York and London (1980)

50. Vainberg, E., Kazak, I.A., Kurozaev, V.P.: Reconstruction of the internal threedimensional structure of objects based on real-time integral projections. Soviet Journal of Nondestructive Testing 17, 415-423 (1981) 